\title{
Conformal Traceless Decomposition of Lagrange Multiplier Modified Hořava-Lifshitz Gravity
}

\author{
J. Klusoň \\ Department of Theoretical Physics and Astrophysics \\ Faculty of Science, Masaryk University \\ Kotlár̆ská 2, 611 37, Brno \\ Czech Republic \\ E-mail: klu@physics.muni.cz
}

\begin{abstract}
We introduce conformal traceless decomposition in Lagrange Multiplier modified RFDiff invariant Hořava-Lifshitz gravity. We perform Hamiltonian analysis of given action and determine the action for the physical degrees of freedom.
\end{abstract}

KEYWORDS: Hořava-Lifshitz gravity. 


\section{Contents}

1. Introduction and Summary 1

2. Brief Review of Lagrange Multiplier Modified HL Gravity 3

3. Conformal Traceless Decomposition

\section{Introduction and Summary}

In 2009 Petr Hořava formulated new proposal of quantum theory of gravity (now known as Hořava-Lifshitz gravity (HL gravity)) that is power counting renormalizable [1, 2, 3] that is also expected that it reduces do General Relativity in the infrared (IR) limit ${ }^{1}$. The HL gravity is based on an idea that the Lorentz symmetry is restored in IR limit of given theory while it is absent in its high energy regime. For that reason Hořava considered systems whose scaling at short distances exhibits a strong anisotropy between space and time,

$$
\mathbf{x}^{\prime}=l \mathbf{x}, \quad t^{\prime}=l^{z} t
$$

In order to have power counting renormalizable theory we have to demand that $z \geq 3$ in $(3+1)$ dimensional space-time. It turns out however that the symmetry group of given theory is reduced from the full diffeomorphism invariance of General Relativity to the foliation preserving diffeomorphism

$$
x^{i}=x^{i}+\zeta^{i}(t, \mathbf{x}), \quad t^{\prime}=t+f(t) .
$$

Due to the fact that the diffeomorphism is restricted (1.2) one more degree of freedom appears that is a spin -0 graviton. The existence of this mode could have very significant consequences either for the consistency of given theory or for the phenomenological applications of HL gravity. For that reason it would be desirable to formulate HL gravity where the number of the physical degrees of freedom is the same as in case of General Relativity. Such a proposal was formulated by Hořava and Malby-Thompson in [15] in the context of the projectable HL gravity ${ }^{2}$. Their construction is based on an extension of the foliation preserving diffeomorphism in such a way that the theory is invariant under additional local $U(1)$ symmetry. The resulting theory is known as non-relativistic covariant theory of gravity. It was shown in 15, 16] that the presence of this new symmetry implies that the spin-0 graviton becomes non-propagating and the spectrum of the linear fluctuations around the background solution coincides with the fluctuation spectrum of General Relativity.

\footnotetext{
${ }^{1}$ For review and extensive list of references, see [4, 9, 10, 11].

${ }^{2}$ See also 23] and [24]
} 
It is also well known that General Relativity contains large number of symmetries. Fixing all these symmetries we find that there are only two physical degrees of freedom left. Then we can ask the question whether it is possible to formulate the action for these physical degrees of freedom that is not based on the principle of covariance of the action under general diffeomorphism. The construction of such an action was proposed recently in two very interesting papers [18, 17]. The basic idea presented there was to perform the conformal traceless decomposition of the gravitational field [6] so that we have one degrees of freedom corresponding to the scale factor of the metric while we have five degrees of freedom of the metric that is restricted to have unit determinant. Then it was shown in [18 that by gauge fixing of the Hamiltonian constraint one can eliminate the scale factor together with the conjugate momenta. As a result we obtain the action for five degrees of freedom that is invariant under spatial diffeomorphism where now Hamiltonian is determined by the solving of the Hamiltonian constraint of the General Relativity for the momentum conjugate to the scale factor. This analysis was further generalized in a very nice paper in [17] where the starting point was the action for the five physical degrees of freedom where it is required that given theory is invariant under spatial diffeomorphism. In other words we demand that the constraints that are generators of the spatial diffeomorphism are the first class constraints. We also have to require that these generators are preserved during the time evolution of the system. Then the requirement of the closure of the algebra of the Poisson brackets of these constraints together with the requirement of their time preservation determines the form of the Hamiltonian and the form of these constraints. When it is presumed that these constraints depend on partial derivatives of $g_{i j}$ trough the scalar curvature we find that the original General Relativity action is reproduced.

The goal of this paper is to formulate HL gravity for the gravitational physical degrees of freedom only in the similar way as in [18]. To do this we start with another version of HL gravity that has the correct number of physical degrees of freedom and which is known as Lagrange multiplier modified HL gravity [7]. This model is based on the formulation of the HL gravity with reduced symmetry group known as restricted-foliation-preserving Diff (RFDiff) HL gravity [12, 7]. This is the theory that is invariant under following symmetries

$$
t^{\prime}=t+\delta t, \delta t=\text { const }, \quad x^{i}=x^{i}+\zeta^{i}(\mathbf{x}, t) .
$$

The characteristic property of Lagrange multiplier modified HL gravity is an absence of the Hamiltonian constraint [13] and also presence of the additional constraint which changes the constraint structure of given theory so that the number of physical degrees of freedom is the same as in the case of General Relativity. Then in order to separate physical degrees of freedom of HL gravity we perform conformal traceless decomposition of the gravitational field, following [6, 8]. In this procedure we introduce new additional scalar field with additional symmetry so that the number of physical degrees of freedom is the same. Performing Hamiltonian analysis we also identify two second class constraints that, together with the gauge fixing scaling symmetry allow us to find Hamiltonian for the physical degrees of freedom, at least in principle. These physical degrees of freedom are metric with unit determinant and conjugate traceless momenta so that the number of physical degrees 
of freedom is the same as in the case of General Relativity. On the other hand there are also important differences. Since this theory arises from the theory with the complicated second class constraints we find that there is a very complicated symplectic structure on the phase space of the physical degrees of freedom. Secondly, even if we can claim that these second class constraints can be solved in principle we find that their solutions have the form of the non-local perturbative expansions. In other words it is hard to see how such a theory could be useful for some practical computations or even for its path integral formulation.

However we mean that the analysis performed here suggests very interesting direction in further research. The starting point would be the general form of the action for the physical degrees of freedom as was analyzed in [17] where we now presume that the additional term in the diffeomorphism constraint depends either on higher order of scalar curvature as for example $R_{i j} R^{i j}$ or it depends on $R_{i j}$ non-locally. Then we should proceed as in [17] where we demand that the Poisson brackets of the spatial diffeomorphism constraints close on the constraint surface. Then from the requirement of the preservation of these constraints during the time evolution of the system we could determine corresponding Hamiltonian density. We hope to return to this problem in future.

\section{Brief Review of Lagrange Multiplier Modified HL Gravity}

We begin this section with the brief review of the Lagrange multiplier modified RFDiff invariant HL gravity, for more detailed treatment see [7]. RFDiff invariant Hořava-Lifshitz gravity was introduced in [12], see also [13]. In [7] this action was extended by introducing Lagrange multiplier term that ensures that the spatial curvature is constant. Explicitly,Lagrange multiplier modified RFDiff HL gravity has the form

$$
S=\frac{1}{\kappa^{2}} \int d t d^{3} \mathbf{x} \sqrt{h}\left(\tilde{K}_{i j} \mathcal{G}^{i j k l} \tilde{K}_{k l}-\mathcal{V}(h)+\mathcal{G}[R] \mathcal{A}\right),
$$

where $\mathcal{G}[R]=R-\Omega$, where $\Omega$ is constant, $\mathcal{A}$ is Lagrange multiplier that transforms as scalar

$$
\mathcal{A}^{\prime}\left(t^{\prime}, \mathbf{x}^{\prime}\right)=\mathcal{A}(t, \mathbf{x})
$$

under (1.3). Further, $\tilde{K}_{i j}$ introduced in (2.1) is modified extrinsic curvature

$$
\tilde{K}_{i j}=\frac{1}{2}\left(\partial_{t} h_{i j}-\nabla_{i} N_{j}-\nabla_{j} N_{i}\right)
$$

that differs from the standard extrinsic curvature by absence of the lapse $N(t)$. Further the generalized De Witt metric $\mathcal{G}^{i j k l}$ is defined as

$$
\mathcal{G}^{i j k l}=\frac{1}{2}\left(h^{i k} h^{j l}+h^{i l} h^{j k}\right)-\lambda h^{i j} h^{k l},
$$

where $\lambda$ is a real constant that in case of General Relativity is equal to one. Finally $\mathcal{V}(h)$ is a general function of $h_{i j}$ and its covariant derivative. The analysis performed in [7] showed that this theory possesses the same number of physical degrees of freedom as General Relativity. For that reason we mean that this action is a good candidate for the conformal traceless decomposition of the gravitational field and possible identification of the physical degrees of freedom of HL gravity. 


\section{Conformal Traceless Decomposition}

The conformal-traceless decomposition of the gravitational field was firstly performed in [5] in its initial value problem ${ }^{3}$. In order to implement conformal-traceless decomposition we follow [6] and define $h_{i j}$ and $\tilde{K}_{i j}$ as

$$
h_{i j}=\phi^{4} g_{i j}, \quad \tilde{K}_{i j}=\phi^{-2} A_{i j}+\frac{1}{3} \phi^{4} g_{i j} \tau .
$$

We see that this definition is redundant since the multiple of the fields $g_{i j}, \phi, A_{i j}, \tau$ give the same physical metric $h_{i j}$ and modified extrinsic curvature $\tilde{K}_{i j}$. In fact, we see that the decomposition (3.1) is invariant under the conformal transformation

$$
\begin{aligned}
g_{i j}^{\prime}(\mathbf{x}, t) & =\Omega^{4}(\mathbf{x}, t) g_{i j}(\mathbf{x}, t), \quad \phi^{\prime}(\mathbf{x}, t)=\Omega^{-1}(\mathbf{x}, t) \phi(\mathbf{x}, t) \\
A_{i j}^{\prime}(\mathbf{x}, t) & =\Omega^{-2}(\mathbf{x}, t) A_{i j}(\mathbf{x}, t), \quad \tau^{\prime}(\mathbf{x}, t)=\tau(\mathbf{x}, t)
\end{aligned}
$$

We also see that (3.1) is invariant under following transformation

$$
\tau^{\prime}(\mathbf{x}, t)=\tau(\mathbf{x}, t)+\zeta(\mathbf{x}, t), \quad A_{i j}^{\prime}(\mathbf{x}, t)=A_{i j}(\mathbf{x}, t)-\frac{1}{3} \zeta(\mathbf{x}, t) \phi^{6} g_{i j}(\mathbf{x}, t) .
$$

Clearly the gauge fixing of these symmetries we can eliminate $\tau$ and $\phi$.

In order to perform the Hamiltonian analysis of the conformal decomposition of the action (2.1) we firstly rewrite the action (2.1) to its Hamiltonian form. To do this we introduce the conjugate momenta

$$
P^{i j}=\frac{\delta S}{\delta \partial_{t} h_{i j}}=\frac{1}{\kappa^{2}} \sqrt{h} \mathcal{G}^{i j k l} \tilde{K}_{k l}, \quad P_{i}=\frac{\delta S}{\delta \partial_{t} N^{i}}=0, \quad P_{\mathcal{A}}=\frac{\delta S}{\delta \partial_{t} \mathcal{A}} \approx 0 .
$$

Then we easily determine corresponding Hamiltonian

$$
H=\int d^{3} \mathbf{x}\left(\partial_{t} h_{i j} P^{i j}-\mathcal{L}\right)=\int d^{3} \mathbf{x}\left(\mathcal{H}_{T}^{\prime}+N^{i} \mathcal{H}_{i}^{\prime}\right)
$$

where

$$
\mathcal{H}_{T}^{\prime}=\frac{\kappa^{2}}{\sqrt{h}} P^{i j} \mathcal{G}_{i j k l} P^{k l}+\sqrt{g} \mathcal{V}(h)-\sqrt{h} \mathcal{A} \mathcal{G}(R), \quad \mathcal{H}_{i}^{\prime}=-2 h_{i j} \nabla_{k} P^{j k} .
$$

Using the Hamiltonian and the corresponding canonical variables we write the action (2.1) as

$$
S=\int d t L=\int d t d^{3} \mathbf{x}\left(P^{i j} \partial_{t} h_{i j}-\mathcal{H}\right)=\int d t d^{3} \mathbf{x}\left(P^{i j} \partial_{t} h_{i j}-N \mathcal{H}_{T}^{\prime}-N^{i} \mathcal{H}_{i}^{\prime}\right)
$$

Then we insert the decomposition (3.1) into the definition of the canonical momenta $P^{i j}$

$$
P^{i j}=\frac{1}{\kappa^{2}} \sqrt{g}\left(\phi^{-4} \tilde{\mathcal{G}}^{i j k l} A_{k l}+\frac{1}{3} \phi^{2} \tau \tilde{\mathcal{G}}^{i j k l} g_{k l}\right)
$$

\footnotetext{
${ }^{3}$ For review and extensive list of references, see [14].
} 
where the metric $\tilde{\mathcal{G}}^{i j k l}$ is defined as

$$
\tilde{\mathcal{G}}^{i j k l}=\frac{1}{2}\left(g^{i k} g^{j l}+g^{i l} g^{j k}\right)-\lambda g^{i j} g^{k l}, \quad \mathcal{G}^{i j k l}=\phi^{-8} \tilde{\mathcal{G}}^{i j k l} .
$$

Note that $\tilde{\mathcal{G}}^{i j k l}$ has the inverse

$$
\tilde{\mathcal{G}}_{i j k l}=\frac{1}{2}\left(g_{i k} g_{j l}+g_{i l} g_{j k}\right)-\frac{\lambda}{3 \lambda-1} g_{i j} g_{k l}, \quad \tilde{\mathcal{G}}_{i j k l}=\phi^{8} \mathcal{G}_{i j k l} .
$$

Using (3.8) and (3.1) we rewrite $P^{i j} \partial_{t} h_{i j}$ into the form

$$
\begin{aligned}
P^{i j} \partial_{t} h_{i j} & =\left(\frac{1}{\kappa^{2}} \sqrt{g} \tilde{\mathcal{G}}^{i j k l} A_{k l}+\frac{\sqrt{g}}{3 \kappa^{2}} \phi^{6}(1-3 \lambda) \tau g^{i j}\right) \partial_{t} g_{j i}+ \\
& +\left(\frac{4}{\kappa^{2}} \sqrt{g} \phi^{-1} A_{k l} g^{k l}(1-3 \lambda)+\frac{4 \sqrt{g}}{\kappa^{2}}(1-3 \lambda) \phi^{5} \tau\right) \partial_{t} \phi
\end{aligned}
$$

We see that it is natural to identify the expression in the parenthesis with momentum $\pi^{i j}$ conjugate to $g_{i j}$ and $p_{\phi}$ conjugate to $\phi$ respectively

$$
\begin{aligned}
\pi^{i j} & =\frac{1}{\kappa^{2}} \sqrt{g} \tilde{\mathcal{G}}^{i j k l} A_{k l}+\frac{\sqrt{g}}{3 \kappa^{2}}(1-3 \lambda) \phi^{6} \tau g^{i j}, \\
p_{\phi} & =\frac{4}{\kappa^{2}} \sqrt{g} \phi^{-1} A_{i j} g^{j i}(1-3 \lambda)+\frac{4 \sqrt{g}}{\kappa^{2}}(1-3 \lambda) \phi^{5} \tau .
\end{aligned}
$$

Then using (3.12) we obtain following primary constraint

$$
\Sigma_{D}: p_{\phi} \phi-4 \pi^{i j} g_{j i}=0 .
$$

As we will see below this is the constraint that generates conformal transformation of the dynamical fields. Further, using (3.12) we find the relation between $P^{i j}$ and $\pi^{i j}$ in the form

$$
P^{i j}=\phi^{-4} \pi^{i j} .
$$

Then we find that the kinetic term in $\mathcal{H}_{T}$ takes the form

$$
\frac{\kappa^{2}}{\sqrt{h}} P^{i j} \mathcal{G}_{i j k l} P^{k l}=\frac{\kappa^{2} \phi^{-6}}{\sqrt{g}} \pi^{i j} \tilde{\mathcal{G}}_{i j k l} \pi^{k l}
$$

As the next step we introduce the decomposition (3.1) into the contribution $\int d^{3} \mathbf{x} N^{i} \mathcal{H}_{i}^{\prime}$. Using the relation between Levi-Civita connections evaluated with the metric components $h_{i j}$ and $g_{i j}$

$$
\Gamma_{i j}^{k}(h)=\Gamma_{i j}^{k}(g)+2 \frac{1}{\phi}\left(\partial_{i} \phi \delta_{j}^{k}+\partial_{j} \phi \delta_{i}^{k}-\partial_{l} \phi g^{k l} g_{i j}\right)
$$

and also if we define $n_{i}$ through the relation $N_{i}=\phi^{4} n_{i}$ we obtain

$$
\int d^{3} \mathbf{x} N^{i} \mathcal{H}_{i}^{\prime}=\int d^{3} \mathbf{x} n^{i} \mathcal{H}_{i}^{\prime \prime}
$$


where

$$
\mathcal{H}_{i}^{\prime \prime}=-2 g_{i k} D_{j} \pi^{j k}+4 \phi^{-1} \partial_{i} \phi g_{k l} \pi^{k l},
$$

where the covariant derivative $D_{i}$ is defined using the Levi-Civita connection $\Gamma_{i j}^{k}(g)$. Observe that with the help of the constraint $\Sigma_{D}$ we can write the constraint $\mathcal{H}_{i}^{\prime \prime}$ as

$$
\mathcal{H}_{i}^{\prime \prime}=-2 g_{i k} D_{j} \pi^{j k}+\partial_{i} \phi p_{\phi}-4 \phi^{-1} \partial_{i} \phi \Sigma_{D} \equiv \hat{\mathcal{H}}_{i}-4 \phi^{-1} \partial_{i} \phi \Sigma_{D}
$$

so that we see that it is natural to identify $\hat{\mathcal{H}}_{i}$ as an independent constraint. In fact, we will see that the smeared form of this constraint generates the spatial diffeomorphism.

Finally we should proceed to the analysis of the spatial curvature and generally the whole potential term $\mathcal{V}$. Note that this is the function of the covariant derivative, $R$ and $R_{i j}$. Using the following formulas

$$
\begin{aligned}
R_{i j}[h] & =R_{i j}[g]+\frac{6}{\phi^{2}} D_{i} \phi D_{j} \phi-\frac{2}{\phi} D_{i} D_{j} \phi-2 \frac{g_{i j}}{\phi} D_{k}\left[g^{k l} D_{l} \phi\right]-\frac{2}{\phi^{2}} g_{i j} D_{k} \phi g^{k l} D_{l} \phi, \\
R[h] & =\phi^{-4}\left[R[g]-\frac{8}{\phi} g^{i j} D_{i} D_{j} \phi\right],
\end{aligned}
$$

Then using also the relation between Levi-Civita connections evaluated on $h$ and $g$ we find that the potential term is generally function of $\phi$ and $g$ whose explicit form is not needed here. As a result we find the action in the form

$$
\begin{aligned}
S & =\int d t d^{3} \mathbf{x}\left(\pi^{i j} \partial_{t} g_{i j}+p_{\phi} \partial_{t} \phi-n^{i} \hat{\mathcal{H}}_{i}-\frac{\kappa^{2} \phi^{-6}}{\sqrt{g}} \pi^{i j} \tilde{\mathcal{G}}_{i j k l} \pi^{k l}-\sqrt{g} \phi^{6} \mathcal{V}(\phi, h)+\right. \\
& \left.+\sqrt{g} \phi^{6} \mathcal{A} \mathcal{G}\left(\phi^{-4} R[g]-\frac{8}{\phi^{5}} g^{i j} D_{i} D_{j} \phi\right)-\lambda \Sigma_{D}\right),
\end{aligned}
$$

where we included the primary constraint $\Sigma_{D}$ multiplied by the Lagrange multiplier $\lambda$.

Now we can proceed to the Hamiltonian analysis of the conformal decomposition of the gravitational field given by the action (3.21). Clearly we have following primary constraints

$$
\pi_{i} \approx 0, \pi_{\mathcal{A}} \approx 0, \quad \Sigma_{D} \approx 0
$$

where $\pi_{i}$ and $\pi_{\mathcal{A}}$ are momenta conjugate to $n^{i}$ and $\mathcal{A}$ with following non-zero Poisson brackets

$$
\left\{n^{i}(\mathbf{x}), \pi_{j}(\mathbf{y})\right\}=\delta_{j}^{i} \delta(\mathbf{x}-\mathbf{y}), \quad\left\{\mathcal{A}(\mathbf{x}), \pi_{\mathcal{A}}(\mathbf{y})\right\}=\delta(\mathbf{x}-\mathbf{y}) .
$$

Further, the preservation of the primary constraints $\pi_{i}$ and $\pi_{\mathcal{A}}$ implies following secondary ones

$$
\hat{\mathcal{H}}_{i} \approx 0, \Phi_{1} \equiv \frac{1}{\kappa^{2}} \sqrt{g} \phi^{6} \mathcal{G} \approx 0 .
$$

Now we should analyze the requirement of the preservation of the primary constraint $\Sigma_{D}$ during the time evolution of the system. First of all the explicit calculations give

$$
\left\{\Sigma_{D}(\mathbf{x}), g_{i j}(\mathbf{y})\right\}=4 g_{i j}(\mathbf{x}) \delta(\mathbf{x}-\mathbf{y})
$$




$$
\begin{aligned}
\left\{\Sigma_{D}(\mathbf{x}), \pi^{i j}(\mathbf{y})\right\} & =-4 \pi^{i j}(\mathbf{x}) \delta(\mathbf{x}-\mathbf{y}) \\
\left\{\Sigma_{D}(\mathbf{x}), \phi(\mathbf{y})\right\} & =-\phi(\mathbf{x}) \delta(\mathbf{x}-\mathbf{y}) \\
\left\{\Sigma_{D}(\mathbf{x}), p_{\phi}(\mathbf{y})\right\} & =\phi(\mathbf{x}) \delta(\mathbf{x}-\mathbf{y})
\end{aligned}
$$

using the canonical Poisson brackets

$$
\left\{g_{i j}(\mathbf{x}), \pi^{k l}(\mathbf{y})\right\}=\frac{1}{2}\left(\delta_{i}^{k} \delta_{j}^{l}+\delta_{i}^{l} \delta_{j}^{k}\right) \delta(\mathbf{x}-\mathbf{y}), \quad\left\{\phi(\mathbf{x}), p_{\phi}(\mathbf{y})\right\}=\delta(\mathbf{x}-\mathbf{y}) .
$$

It turns out that it is useful to introduce the smeared forms of the constraints $\hat{\mathcal{H}}_{i}, \Sigma_{D}$

$$
\mathbf{T}_{S}\left(N^{i}\right)=\int d^{3} \mathbf{x} N^{i} \hat{\mathcal{H}}_{i}, \quad \mathbf{D}(M)=\int d^{3} \mathbf{x} M \Sigma_{D}
$$

where $N^{i}$ and $M$ are smooth functions on $\mathbf{R}^{3}$. Then using (3.25) and also

$$
\left\{\Sigma_{D}(\mathbf{x}), \Gamma_{i j}^{k}(\mathbf{y})\right\}=2 \delta_{j}^{k} \partial_{y^{i}} \delta(\mathbf{x}-\mathbf{y})+2 \delta_{i}^{k} \partial_{y^{j}} \delta(\mathbf{x}-\mathbf{y})-2 g^{k l}(\mathbf{y}) \partial_{y^{l}} \delta(\mathbf{x}-\mathbf{y}) g_{i j}(\mathbf{y})
$$

we easily find that

$$
\left\{\mathbf{D}(M), \mathcal{H}_{T}^{\prime}(\mathbf{y})\right\}=0
$$

where

$$
\mathcal{H}_{T}^{\prime}=\frac{\kappa^{2} \phi^{-6}}{\sqrt{g}} \pi^{i j} \tilde{\mathcal{G}}_{i j k l} \pi^{k l}+\sqrt{g} \phi^{6} \mathcal{V}(\phi, g) .
$$

To proceed further we use following Poisson brackets

$$
\begin{aligned}
\left\{\mathbf{T}_{S}\left(N^{i}\right), g_{i j}(\mathbf{x})\right\} & =-N^{k} \partial_{k} g_{i j}(\mathbf{x})-\partial_{i} N^{k} g_{k j}(\mathbf{x})-g_{i k} \partial_{j} N^{k}(\mathbf{x}), \\
\left\{\mathbf{T}_{S}\left(N^{i}\right), \pi^{i j}(\mathbf{x})\right\} & =-\partial_{k}\left(N^{k} \pi^{i j}\right)(\mathbf{x})+\partial_{k} N^{i} \pi^{k j}(\mathbf{x})+\pi^{i k} \partial_{k} N^{j}(\mathbf{x}), \\
\left\{\mathbf{T}_{S}\left(N^{i}\right), \phi(\mathbf{x})\right\} & =-N^{i} \partial_{i} \phi(\mathbf{x}), \\
\left\{\mathbf{T}_{S}\left(N^{i}\right), p_{\phi}(\mathbf{x})\right\} & =-\partial_{i}\left(N^{i} p_{\phi}\right)(\mathbf{x})
\end{aligned}
$$

and hence it is easy to see that

$$
\left\{\mathbf{T}_{S}\left(N^{i}\right), \Sigma_{D}(\mathbf{x})\right\}=-N^{i} \partial_{i} \Sigma_{D}(\mathbf{x})-\partial_{i} N^{i} \Sigma_{D}(\mathbf{x})
$$

that together with $(3.29)$ implies that $\Sigma_{D} \approx 0$ is the first class constraint.

Now we proceed to the analysis of the preservation of the secondary constraints $\hat{\mathcal{H}}_{i} \approx 0$ and $\Phi_{1} \approx 0$. Note that the total Hamiltonian takes the form

$$
H_{T}=\int d^{3} \mathbf{x}\left(\mathcal{H}_{T}^{\prime}+\lambda \Sigma_{D}+n^{i} \hat{\mathcal{H}}_{i}+\gamma p_{\mathcal{A}}+\Gamma_{I} \Phi_{1}\right)
$$

where $\gamma$ is the Lagrange multiplier corresponding to the constraint $p_{\mathcal{A}}$ while $\Gamma_{I}$ is the Lagrange multiplier corresponding to the constraint $\Phi_{1} \approx 0$. 
In case of $\hat{\mathcal{H}}_{i}$ we find following Poisson brackets

$$
\left\{\hat{\mathcal{H}}_{i}(\mathbf{x}), \hat{\mathcal{H}}_{j}(\mathbf{y})\right\}=\hat{\mathcal{H}}_{j}(\mathbf{x}) \frac{\partial}{\partial x^{i}} \delta(\mathbf{x}-\mathbf{y})-\hat{\mathcal{H}}_{i}(\mathbf{y}) \frac{\partial}{\partial y^{j}} \delta(\mathbf{x}-\mathbf{y})
$$

which implies that the smeared form of the diffeomorphism constraints takes the familiar form

$$
\left\{\mathbf{T}_{S}\left(N^{i}\right), \mathbf{T}_{S}\left(M^{j}\right)\right\}=\mathbf{T}_{S}\left(N^{j} \partial_{j} M^{i}-M^{j} \partial_{j} N^{i}\right) .
$$

Further using (3.31) we easily find

$$
\begin{array}{r}
\left\{\mathbf{T}_{S}\left(N^{i}\right), \mathcal{H}_{T}^{\prime}(\mathbf{x})\right\}=-\partial_{i} N^{i} \mathcal{H}_{T}^{\prime}(\mathbf{x})-N^{i} \partial_{i} \mathcal{H}_{T}^{\prime}(\mathbf{x}) \\
\left\{\mathbf{T}_{S}\left(N^{i}\right), \Phi_{1}(\mathbf{x})\right\}=-\partial_{i} N^{i} \Phi_{1}(\mathbf{x})-N^{i} \partial_{i} \Phi_{1}(\mathbf{x})
\end{array}
$$

that implies that $\hat{\mathcal{H}}_{i}$ are the first class constraints that are preserved during the time evolution of the system. Finally we analyze the time evolution of the constraint $\Phi_{1} \approx 0$. Using following formulas

$$
\begin{aligned}
\left\{R(\mathbf{x}), \pi^{i j}(\mathbf{y})\right\} & =-R^{i j}(\mathbf{x}) \delta(\mathbf{x}-\mathbf{y})+D^{i} D^{j} \delta(\mathbf{x}-\mathbf{y})-g^{i j} D_{k} D^{k} \delta(\mathbf{x}-\mathbf{y}) \\
\left\{\Gamma_{i j}^{k}(\mathbf{x}), \pi^{m n}(\mathbf{y})\right\} & =\frac{1}{4} g^{k p}\left[D_{i} \delta\left(\delta_{j}^{m} \delta_{p}^{n}+\delta_{j}^{n} \delta_{p}^{m}\right) \delta(\mathbf{x}-\mathbf{y})+\right. \\
& \left.+D_{j}\left(\delta_{p}^{m} \delta_{i}^{n}+\delta_{p}^{n} \delta_{i}^{m}\right) \delta(\mathbf{x}-\mathbf{y})-D_{p}\left(\delta_{i}^{m} \delta_{j}^{n}+\delta_{i}^{n} \delta_{j}^{m}\right) \delta(\mathbf{x}-\mathbf{y})\right]
\end{aligned}
$$

we find that the time derivative of $\Phi_{1}$ is equal to

$$
\begin{aligned}
\partial_{t} \Phi_{1} & =\left\{\Phi_{1}, H_{T}\right\} \approx \\
& -\frac{2 \kappa^{2}}{\phi^{4} \sqrt{g}}\left(R_{i j} \pi^{i j}-\frac{\lambda}{3 \lambda-1} R \pi\right)+ \\
& +\frac{2 \kappa^{2} \phi^{2}}{\sqrt{g}} D_{k} D_{l}\left[\phi^{-6} \pi^{k l}\right]+\frac{2 \kappa^{2} \phi^{2}}{\sqrt{g}} \frac{(1-\lambda)}{3 \lambda-1} D_{k} D^{k}\left[\phi^{-6} \pi\right]- \\
& -16 \frac{\kappa^{2}}{\phi^{5} \sqrt{g}}\left(\pi^{i j}-\frac{\lambda}{3 \lambda-1} g^{i j} \pi\right) D_{i} D_{j} \phi+ \\
& +\frac{16 \phi \kappa^{2}}{\sqrt{g}} D_{i} \phi D_{j}\left[\phi^{-6} \pi^{i j}\right]-\frac{8 \kappa^{2} \phi}{\sqrt{g}} \frac{2 \lambda-1}{3 \lambda-1} D_{i} \phi g^{i j} D_{j}\left[\phi^{-6} \pi\right] \equiv \Phi_{2},
\end{aligned}
$$

where $\Phi_{2}$ is an additional constraint that has to be imposed on the system. Following [19, 20, 21] we include the constraint $\Phi_{2}$ into the definition of the total Hamiltonian that now has the form

$$
\begin{aligned}
H_{T} & =\int d^{3} \mathbf{x}\left(\mathcal{H}_{T}^{\prime}-\sqrt{g} \phi^{6} \mathcal{A}\left(\phi^{-4} R[g]-8 \phi^{-5} g^{i j} D_{i} D_{j} \phi-\Omega\right)+\lambda \Sigma_{D}+\right. \\
& \left.+n^{i} \hat{\mathcal{H}}_{i}+\gamma p_{\mathcal{A}}+\Gamma_{I} \Phi_{1}+\Gamma_{I I} \Phi_{2}\right)
\end{aligned}
$$


Now we should again check the stability of all constraints. It is easy to see that the primary constraints together with $\mathbf{T}_{S}\left(N^{i}\right)$ are preserved while the time evolution of the constraint $\Phi_{1} \approx 0$ is equal to

$$
\begin{aligned}
\partial_{t} \Phi_{1} & =\left\{\Phi_{1}, H_{T}\right\} \approx \int d^{3} \mathbf{x}\left(\Gamma^{I I}(\mathbf{x})\left\{\Phi_{1}, \Phi_{2}(\mathbf{x})\right\}\right) \approx \\
& \approx \int d^{3} \mathbf{x} \Gamma^{I I}(\mathbf{x})\left\{\Phi_{1}, \Phi_{2}(\mathbf{x})\right\}=0 .
\end{aligned}
$$

As follows from the explicit form of the constraints $\Phi_{1,2}$ we have

$$
\left\{\Phi_{1}(\mathbf{x}), \Phi_{2}(\mathbf{y})\right\} \neq 0 .
$$

Then we find that the equation $(3.40)$ gives $\Gamma^{I I}=0$. In the same way the requirement of the preservation of the constraint $\Phi_{2}$ implies

$$
\partial_{t} \Phi_{2} \approx \int d^{D} \mathbf{x}\left(\left\{\Phi_{2}, \mathcal{H}_{T}(\mathbf{x})\right\}+\Gamma^{I}(\mathbf{x})\left\{\Phi_{2}, \Phi_{1}(\mathbf{x})\right\}\right)=0
$$

Using the fact that $\left\{\Phi_{2}, \mathcal{H}_{T}(\mathbf{x})\right\} \neq 0$ and also the equation (3.41) we see that (3.42) can be solved for $\Gamma^{I}$. In fact, (3.41) shows that $\Phi_{1}$ and $\Phi_{2}$ are the second class constraints. We also see from the previous analysis that no additional constraints have to be imposed on the system.

In order to find the action for the physical degrees of freedom we have to finally fix the gauge symmetry generated by $\Sigma_{D}$. To do this we introduce the gauge fixing function

$$
\Phi_{G . F .}=\sqrt{g}-1 .
$$

It is easy to see that there is non-zero Poisson bracket between $\Phi_{G . F}$. and $\Sigma_{D}$ so that they are the second class constraints. In summary we have following collection of the second class constraints

$$
\Phi_{1}=0, \Phi_{2}=0, \Sigma_{D}=0, \Phi_{G . F .}=0 .
$$

The goal is to eliminate some degrees of freedom from these constraints, at least in principle. In fact, from $\Phi_{1}$, which is version of Lichnerowitz-York equation [22], we express $\phi$ as

$$
\phi=\frac{1}{8} \nabla^{-1}\left(\phi R[g]-\phi^{5} \Omega\right) .
$$

where $\nabla^{-1}$ is inverse operator to $g^{i j} D_{i} D_{j}$. We can solve the equation above perturbatively around some constant $\phi_{0}$. Further, from $\Sigma_{D}$ we express $p_{\phi}$. Finally, $\Phi_{G . F}$. reduces number of degrees of freedom in $g$ to be equal to five and from $\Phi_{2}$ we express $\pi$ as the function of remaining degrees of freedom. In summary, the physical degrees of freedom of Lagrange multiplier modified HL gravity are

$$
g_{i j}, \quad \sqrt{g}=1, \quad \tilde{\pi}^{i j}, g_{i j} \tilde{\pi}^{j i}=0 .
$$


Note that there are three first class constraints $\hat{\mathcal{H}}_{i}$ so that by gauge fixing these constraints we should eliminate remaining three degrees of freedom in $g_{i j}$. In other words the physical content of given theory is the same as in General Relativity.

From the previous analysis we see that $\phi$ is a non-local function of $R$. The same situation also occurs in case of $\pi$ so that in principle $\hat{\mathcal{H}}_{i}$ has the form

$$
\hat{\mathcal{H}}_{i}=-2 g_{i k} \nabla_{j} \tilde{\pi}^{k j}+p_{\phi}\left(\nabla^{-1} f(g, \pi)\right) \nabla_{i} \phi\left(\nabla^{-1} R(g)\right)-\frac{1}{3} \nabla_{i} \pi\left(\nabla^{-1}, \tilde{\pi}, g\right) .
$$

By definition they are the first class constraints which however depends non-locally on the canonical variables. We would like to stress that this result can be considered as the generalization of the analysis performed in 17, 18] to the case of HL gravity. In more details, papers cited above were devoted to the construction of the action for the physical modes of the gravitational fields only that are the metric that obeys the condition $\sqrt{g}=1$ and the conjugate momenta $\tilde{\pi}^{i j}$ that are traceless. In order to have the right number of physical degrees of freedom this action should be invariant under spatial diffeomorphism. Explicitly, the action studied there has the form

$$
S=\int d t d^{3} \mathbf{x}\left(\dot{g}_{i j} \tilde{\pi}^{i j}-\pi_{H}-N^{i} \tilde{\mathcal{H}}_{i}\right)
$$

where

$$
\tilde{\mathcal{H}}_{i}=-2 g_{i j} \nabla_{k} \tilde{\pi}^{j k}-\nabla_{i} \pi_{K},
$$

where $\pi_{K}$ is arbitrary function that has to be determined in such a way that $\tilde{\mathcal{H}}_{i}$ are the first class constraints. However when we restrict to the case when this function depends on the partial derivatives of $g_{i j}$ through the scalar curvature $R$ we find that the functions $\pi_{N}$ and $\pi_{K}$ are uniquely determined and leads to the General Relativity action. Note also that the symplectic structure used in given paper has the standard form

$$
\left\{g_{i j}, \tilde{\pi}^{k l}\right\}=\frac{1}{2}\left(\delta_{i}^{k} \delta_{j}^{l}+\delta_{i}^{l} \delta_{j}^{k}\right)-\frac{1}{3} g_{i j} g^{k l} .
$$

In case of Lagrange multiplier modified HL gravity the situation is more involved. The symplectic structure is determined by the Poisson brackets of the second class constraints $\Phi_{1}, \Phi_{2}$ which is rather complicated. More precisely, let us denote all second class constraints as $\Phi_{A}=\left(\Sigma_{D}, \Phi_{G . F .}, \Phi_{1}, \Phi_{2}\right)$. Then the Poisson bracket between the constraints $\Phi_{A}$ can be written as

$$
\left\{\Phi_{A}(\mathbf{x}), \Phi_{B}(\mathbf{y})\right\}=\triangle_{A B}(\mathbf{x}, \mathbf{y}),
$$

where the matrix $\triangle_{A B}$ has following structure

$$
\triangle_{A B}(\mathbf{x}, \mathbf{y})=\left(\begin{array}{cccc}
0 & * & 0 & 0 \\
* & 0 & 0 & * \\
0 & 0 & 0 & * \\
0 & * & * & *
\end{array}\right),
$$

where $*$ denotes non-zero elements that depend on the phase space variables and their derivatives. Now it is easy to see that the Dirac brackets between the canonical variables 
that are defined as

$$
\begin{aligned}
& \left\{g_{i j}(\mathbf{x}), g_{k l}(\mathbf{y})\right\}_{D}=-\int d \mathbf{z} d \mathbf{z}^{\prime}\left\{g_{i j}(\mathbf{x}), \Phi_{A}(\mathbf{z})\right\}\left(\triangle^{-1}\right)^{A B}\left(\mathbf{z}, \mathbf{z}^{\prime}\right)\left\{\Phi_{B}\left(\mathbf{z}^{\prime}\right), g_{k l}(\mathbf{y})\right\}, \\
& \left\{\pi^{i j}(\mathbf{x}), \pi^{k l}(\mathbf{y})\right\}_{D}=-\int d \mathbf{z} d \mathbf{z}^{\prime}\left\{\pi^{i j}(\mathbf{x}), \Phi_{A}(\mathbf{z})\right\}\left(\triangle^{-1}\right)^{A B}\left(\mathbf{z}, \mathbf{z}^{\prime}\right)\left\{\Phi_{B}\left(\mathbf{z}^{\prime}\right), \pi^{k l}(\mathbf{y})\right\}, \\
& \left\{g_{i j}(\mathbf{x}), \pi^{k l}(\mathbf{y})\right\}_{D}=\left\{g_{i j}(\mathbf{x}), \pi^{k l}(\mathbf{y})\right\}- \\
- & \int d \mathbf{z} d \mathbf{z}^{\prime}\left\{g_{i j}(\mathbf{x}), \Phi_{A}(\mathbf{z})\right\}\left(\triangle^{-1}\right)^{A B}\left(\mathbf{z}, \mathbf{z}^{\prime}\right)\left\{\Phi_{B}\left(\mathbf{z}^{\prime}\right), \pi^{k l}(\mathbf{y})\right\}
\end{aligned}
$$

depend on the phase-space variables. Secondly, the resulting action is non-local due the presence of the inverse operator $\nabla^{-1}$.

Even if the action for the physical degrees of freedom of Lagrange multiplier modified HL gravity is rather involved we mean that the results derived here should be considered as the starting point for further research of HL gravity when we generalize the analysis performed in [17, 18] in several different ways. We could start with the action for the physical modes with the symplectic structure given by the equation (3.50) and presume that $\pi_{K}$ depends on $R$ non-locally and try to determine the original action. Another possibility is to consider the case when $\pi_{K}$ in (3.49) depends on $R_{i j} R^{i j}$ and covariant derivatives of $R_{i j}$. We hope to return to these problems in future.

\section{Acknowledgement}

This work was supported by the Grant Agency of the Czech Republic under the grant P201/12/G028.

\section{References}

[1] P. Horava, "Quantum Gravity at a Lifshitz Point," Phys. Rev. D 79 (2009) 084008 [arXiv:0901.3775 [hep-th]].

[2] P. Horava, "Membranes at Quantum Criticality," JHEP 0903 (2009) 020 [arXiv:0812.4287 [hep-th]].

[3] P. Horava, "Quantum Criticality and Yang-Mills Gauge Theory," arXiv:0811.2217 [hep-th].

[4] P. Horava, "General Covariance in Gravity at a Lifshitz Point," arXiv:1101.1081 [hep-th].

[5] J. W. York, Jr., "Conformal 'thin sandwich' data for the initial-value problem," Phys. Rev. Lett. 82 (1999) 1350 [gr-qc/9810051].

[6] J. D. Brown, "Conformal invariance and the conformal-traceless decomposition of the gravitational field," Phys. Rev. D 71 (2005) 104011 [gr-qc/0501092].

[7] J. Kluson, "Lagrange Multiplier Modified Horava-Lifshitz Gravity," Eur. Phys. J. C 71 (2011) 1820 [arXiv:1101.5880 [hep-th]].

[8] J. Kluson, "Hamiltonian Analysis of the Conformal Decomposition of the Gravitational Field," Phys. Rev. D 86 (2012) 084001 [arXiv:1206.5116 [gr-qc]].

[9] A. Padilla, "The good, the bad and the ugly .... of Horava gravity," arXiv:1009.4074 [hep-th]. 
[10] S. Mukohyama, "Horava-Lifshitz Cosmology: A Review," arXiv:1007.5199 [hep-th].

[11] S. Weinfurtner, T. P. Sotiriou and M. Visser, "Projectable Horava-Lifshitz gravity in a nutshell," J. Phys. Conf. Ser. 222, 012054 (2010) [arXiv:1002.0308 [gr-qc]].

[12] D. Blas, O. Pujolas and S. Sibiryakov, "Models of non-relativistic quantum gravity: the good, the bad and the healthy," arXiv:1007.3503 [hep-th].

[13] J. Kluson, "Horava-Lifshitz Gravity And Ghost Condensation," Phys. Rev. D 82 (2010) 124011 [arXiv:1008.5297 [hep-th]].

[14] E. Gourgoulhon, "3+1 Formalism and Bases of Numerical Relativity," arXiv:gr-qc/0703035.

[15] P. Horava and C. M. Melby-Thompson, "General Covariance in Quantum Gravity at a Lifshitz Point," Phys. Rev. D 82 (2010) 064027 [arXiv:1007.2410 [hep-th]].

[16] A. M. da Silva, "An Alternative Approach for General Covariant Horava-Lifshitz Gravity and Matter Coupling," arXiv:1009.4885 [hep-th].

[17] J. Khoury, G. E. J. Miller and A. J. Tolley, "On the Origin of Gravitational Lorentz Covariance," Class. Quant. Grav. 31 (2014) 135011 [arXiv:1305.0822 [hep-th]].

[18] J. Khoury, G. E. J. Miller and A. J. Tolley, "Spatially Covariant Theories of a Transverse, Traceless Graviton, Part I: Formalism," Phys. Rev. D 85 (2012) 084002 [arXiv:1108.1397 [hep-th]].

[19] M. Henneaux and C. Teitelboim, "Quantization of gauge systems," Princeton, USA: Univ. Pr. (1992) $520 p$

[20] J. Govaerts, "The quantum geometer's universe: Particles, interactions and topology," arXiv:hep-th/0207276.

[21] J. Govaerts, "Hamiltonian Quantization And Constrained Dynamics," Leuven, Belgium: Univ. Pr. (1991) 371 p. (Leuven notes in mathematical and theoretical physics, B4)

[22] J. W. York, Jr., "Gravitational degrees of freedom and the initial-value problem," Phys. Rev. Lett. 26 (1971) 1656.

[23] Y. Huang and A. Wang, "Stability, ghost, and strong coupling in nonrelativistic general covariant theory of gravity with $\lambda \neq 1$," Phys. Rev. D 83 (2011) 104012 [arXiv:1011.0739 [hep-th]].

[24] S. Das and S. Ghosh, "Gauge Invariant Extension of Linearized Horava Gravity," Mod. Phys. Lett. A 26 (2011) 2793 [arXiv:1104.1975 [gr-qc]]. 